\title{
Normal inter-limb differences during the straight leg raise neurodynamic test: a cross sectional study
}

Benjamin S Boyd ${ }^{1 *}$ and Philip S Villa ${ }^{2}$

\begin{abstract}
Background: The straight leg raise (SLR) neurodynamic test is commonly used to examine the sensitivity of the lower quarter nervous system to movement. Range of motion during the SLR varies considerably, due to factors such as age, sex and activity level. Knowing intra-individual, inter-limb differences may provide a normative measure that is not influenced by such demographic characteristics. This study aimed to determine normal asymmetries between limbs in healthy, asymptomatic individuals during SLR testing and the relationship of various demographic characteristics.

Methods: The limb elevation angle was measured using an inclinometer during SLR neurodynamic testing that involved pre-positioning the ankle in plantar flexion (PF/SLR) and neutral dorsiflexion (DF/SLR). Phase 1 of the study included 20 participants where the ankle was positioned using an ankle brace replicating research testing conditions. Phase 2 included 20 additional participants where the ankle was manually positioned to replicate clinical testing conditions.

Results: The group average range of motion during PF/SLR was 57.1 degrees (SD: 16.8 degrees) on the left and 56.7 degrees (SD: 17.2 degrees) on the right while during DF/SLR the group average was 48.5 degrees (SD: 16.1 degrees) on the left and 48.9 degrees (SD: 16.4 degrees) on the right. The range of motion during SLR was moderately correlated to weight $(-0.40$ to -0.52$)$, body mass index ( -0.41 to -0.52$)$, sex (0.40 to 0.42$)$ and self-reported activity level ( 0.50 to 0.57 ). Intra-individual differences between limbs for range of motion during PF/SLR averaged 5.0 degrees (SD: 3.5 degrees) (95\% Cl: 3.8 degrees, 6.1 degrees) and during DF/SLR averaged 4.1 degrees (SD: 3.2 degrees) (95\% Cl: 3.1 degrees, 5.1 degrees) but were not correlated with any demographic characteristic. There were no significant differences between Phase 1 and Phase 2.

Conclusions: Overall range of motion during SLR was related to sex, weight, BMI and activity level, which is likely reflected in the high variability documented. We can be $95 \%$ confident that inter-limb differences during SLR neurodynamic testing fall below 11 degrees in $90 \%$ of the general population of healthy individuals. In addition, inter-limb differences were not affected by demographic factors and thus may be a more valuable comparison for test interpretation.
\end{abstract}

\footnotetext{
* Correspondence: bboyd1@samuelmerritt.edu

'Department of Physical Therapy, Samuel Merritt University, 450 30th Street, Oakland, CA 94609, USA

Full list of author information is available at the end of the article
}

\section{Biomed Central}

(c) 2012 Boyd and Villa; licensee BioMed Central Ltd. This is an Open Access article distributed under the terms of the Creative Commons Attribution License (http://creativecommons.org/licenses/by/2.0), which permits unrestricted use, distribution, and reproduction in any medium, provided the original work is properly cited. 


\section{Background}

The straight leg raise (SLR) is a common neurodynamic test used to examine the mechanosensitivity of the lower extremity nervous system in individuals with low back or lower extremity pain [1-4]. Structural differentiation is necessary to determine if symptom provocation and range of motion restrictions are related to neural tissue [5]. Pre-positioning in ankle dorsiflexion compared to plantar flexion is commonly utilized for purposes of structural differentiation during SLR testing $[1,2,6,7]$ and distinguishes the SLR neurodynamic test from a hamstring muscle length test [8]. From here forward SLR will refer to neurodynamic testing. It has been proposed that identification of a "positive," clinically relevant test should include consideration of three components [5]. These components include 1) reproduction of the patient's symptoms in whole or in part, 2) distant movements away from that region altering the symptoms (structural differentiation), and 3) identification of differences in sensory, range of motion or resistance to movement noted between limbs or known norms [5]. Limb elevation angle at the point of a sensory response provides a mobility measurement for the third component. Ideally, normative SLR range of motion in healthy, asymptomatic individuals could be used for comparisons to testing in clinical populations. Unfortunately, when used as a neurodynamic test, normal SLR range of motion is highly variable, averaging from $40^{\circ}$ to $85^{\circ}$ $[1,3,6,9]$. The large degree of variability in range of motion makes valid identification of mobility impairments difficult.

Previous literature has demonstrated that lower extremity range of motion is highly dependent upon multiple factors, such as age, [10-12] sex, $[8,10,11]$ and limb dominance [13]. These demographic factors may explain much of the variability in SLR range of motion but this has yet to be investigated. If these relationships do exist, establishing normative SLR range of motion becomes quite problematic. An alternative approach is to look at symmetry of SLR range of motion within individuals. Previous literature of healthy, asymptomatic individuals found significant intra-individual asymmetries in isolated ankle motions [14] as well as differences between limbs during upper limb neurodynamic testing [15]. Intra-individual, inter-limb differences may be a more useful measure for establishing normative values for SLR testing, as they are less likely to be influenced by other factors such as age, sex, weight and activity level. Providing evidence of normal inter-limb differences during SLR testing in the healthy, asymptomatic population will allow for future comparisons of the differences between the affected limb and unaffected limb in patients experiencing unilateral pain.
The primary aims of this study were to; 1 ) determine the relationship between demographic characteristics and overall SLR range of motion, 2) quantify inter-limb differences during SLR testing in healthy, asymptomatic individuals, under both research conditions and clinical testing conditions. Normal overall SLR range of motion and inter-limb differences are presented with correlations to various demographic characteristics and implications for test interpretation.

\section{Methods}

This cross sectional study included two phases involving SLR neurodynamic testing performed where the ankle was positioned using an ankle brace to replicate research testing conditions (Phase 1) and where the ankle was manually positioned to replicate clinical testing conditions (Phase 2). Each phase included a unique set of 20 healthy, asymptomatic participants ( $\mathrm{n}=40$ total). Participants from Phase 1 were previously reported for purposes of validating the measurement device but data related to inter-limb difference has not been previously published [7].

Participants were recruited from local academic and medical facilities. Inclusion criteria included minimum flexibility requirements of isolated ankle range of motion $>0^{\circ}$ dorsiflexion and $>30^{\circ}$ plantar flexion, full knee extension, and hip flexion $>90^{\circ}$ with the knee flexed. Exclusion criteria included current or recent $(>3$ consecutive days in past 6 months) low back or lower extremity pain, peripheral neuropathy, diabetes mellitus, complex regional pain syndrome, chemical dependence or alcohol abuse, a history of lower extremity nerve trauma, lumbar spine surgeries, or chemotherapy use. The Samuel Merritt University Institutional Review Board approved this study and assured ethical treatment of participants. Prior to testing, written informed consent was obtained. Prior to SLR testing each participant completed the Modified Baecke Questionnaire (MBQ), which is a self-report measure on activity level [16].

\section{Neurological testing}

In order to rule out potential sub-clinical injuries to the nervous system, a segmental neurological examination was performed to confirm that the participants had no signs of conduction loss. In brief, dermatome testing with a 10 gram monofilament was performed in bilateral sensory distributions for segments L3 (medial knee), L4 (medial ankle), L5 (dorsum of foot), S1 (lateral heel) and graded as present or absent. Myotome testing was performed against manual resistance for segments L3 (quadriceps), L4 (tibialis anterior), L5 (extensor hallucis longus), and S1 (fibularis longus and brevis) and graded 
as normal, mild/moderate weakness, severe weakness, or absent. Deep tendon reflexes were performed for L4 (patellar tendon), L5 (semitendinosis tendon), S1 (Achilles tendon) and graded as present or absent. Quantitative sensory testing included vibration perception thresholds (VPT) in bilateral halluces (distal pad) using a $60 \mathrm{~Hz}$ Biothesiometer (Bio-Medical Instruments Company, Newbury, OH, USA) with a scale of 0-50 V. Participants were instructed to indicate the first moment when the vibration was felt as it was slowly turned up from zero and VPT is reported as an average voltage (two trials each limb). Previous literature has identified normal ranges for VPT testing at the halluces as $15 \mathrm{~V}$ or lower $[17,18]$.

\section{SLR testing}

Participants in both phases of testing were placed in a standardized start position which included lying supine on a plinth with a $2.5 \mathrm{~cm}$ thick foam head support. They were positioned with their spine in neutral within the coronal plane with their upper arms resting at their sides. Their lower limbs were positioned in neutral abduction. A hand-held inclinometer was placed against the anterior aspect of the mid-tibia. Measuring limb elevation angle with this device demonstrates excellent reliability (ICC: $0.95-0.98$ ), validity (ICC: 0.88-0.99) and standard error of measurement $\left(0.54-1.22^{\circ}\right)$ when used during the SLR test [7]. The ankle was placed in either dorsiflexion or plantar flexion prior to performing the hip flexion component of the SLR. For phase 1, the ankle was secured in $0^{\circ}$ dorsiflexion or $30^{\circ}$ plantar flexion using an ankle brace and straps (Anatomical Concepts, Inc., Youngstown, OH) [1,2]. For phase 2, the ankle was manually placed in dorsiflexion or plantar flexion to the point of firm resistance as felt by the examiner, as is commonly done clinically. Ankle position was monitored in this phase by use of a twin-axis electrogoniometer (Noraxon, USA, Scottsdale, AZ) that was placed laterally across the ankle with the proximal end parallel to the fibula and the distal end parallel to the $5^{\text {th }}$ metatarsal [3]. The goniometer was held in place with double-sided tape and straps. Dorsiflexion to $0^{\circ}$ was utilized due to the frequency of limitations in ankle dorsiflexion range when the knee is in full extension [19]. Using an electronic trigger held in their dominant hand resting on their abdomen, the participant was instructed to indicate when any sensory response was elicited during the SLR test. The electrogoniometer and hand-held trigger data were acquired at $1000 \mathrm{~Hz}$ using a Myosystem 1400 unit (Noraxon, USA, Scottsdale, AZ).

The SLR consisted of placing the knee in end range extension, determined by the examiner as end range resistance (R2), followed by bringing the limb into hip flexion. Care was taken to avoid movement of the limb in the transverse or coronal planes. The movement was stopped at the first moment any sensory response was indicated, including but not limited to the sensation of stretch, pulling, tension, pain, numbness, or tingling. The use of healthy participants without nerve injury meant that true "positive" neurodynamic test findings (as outlined above) were not possible in this study as there are no "symptoms" to be provoked. However, mobility limitations due to neurogenic sensory responses are common in healthy individuals during neurodynamic testing and should not be interpreted as pathological [15]. Therefore, this study sought to replicate clinical testing procedures by comparing symmetry of range of motion during the two SLR variations taken to the first onset of any sensory response to assist with comparisons to patients with neuropathic pain. The pelvis and lumbar spine were not stabilized to match clinical testing procedures and as these motions, in addition to hip flexion, theoretically contribute to increasing stress on the posterior neural structures of the lower quarter [7]. The limb elevation angle was measured at this point and then the limb was returned to a resting position on the mat [7]. The SLR was performed twice with the ankle in dorsiflexion (DF/SLR) and twice with the ankle in plantar flexion (PF/SLR) with the order randomized to negate the effect of repeated testing. All tests were performed by one examiner with over nine years of clinical and research experience in neurodynamic testing.

\section{Statistical analysis}

The mean of both trials for overall range of motion and inter-limb difference (defined as the absolute difference between right and left limbs) were utilized for statistical analyses using IBM SPSS Statistics, version 19 (IBM Corporation, Somer, NY). Reliability between the two trials was assessed using Intraclass Correlation Coefficient $\left(\mathrm{ICC}_{2,1}\right)$ calculations with $95 \%$ confidence intervals and $95 \%$ limits of agreement [20]. Limits of agreement provide the range within which the expected difference between two repeated measurements within an individual would fall $95 \%$ of the time [20]. Repeated measures analysis of variance (ANOVA) was utilized to compare range of motion differences between limbs and testing conditions. Comparisons were made between Phase 1 and 2 using independent samples t-tests. Correlation coefficients were used to determine relationships between demographic characteristics and range of motion measures. The strength of correlations were determined as 0.00 to $0.25=$ little to no relationship, 0.26 to $0.50=$ fair degree of relationship, 0.51 to $0.75=$ moderate to good relationship, and 0.76 to $1.00=$ good to excellent relationship [21]. Power analysis revealed that with 40 participants and an alpha of 0.05 , we would have $80 \%$ power of detecting a correlation of 0.37 which represents 
at least a "fair" relationship. We did not have adequate power to detect interaction effects. For purposes of generalizing from this sample to the greater population, the upper limit of a tolerance interval (one sided test) was calculated in order to identify a threshold within which $90 \%$ of the values for "normal" inter-limb differences can be expected in the population with $95 \%$ certainty [22]. Alpha was set at 0.05 .

\section{Results}

The demographic characteristics of participants are presented in Table 1. The participants in Phase 1 were slightly older by an average of seven years and rated themselves as slightly more active on the MBQ.

\section{Neurological testing}

All participants had intact sensation in all dermatome levels bilaterally with normal and equal strength bilaterally on myotome testing. Deep tendon reflexes were equal bilaterally in all participants. VPT values were equivalent between limbs and averaged $7.1(2.6 \mathrm{SD}) \mathrm{V}$ which is well within normal ranges $(<15 \mathrm{~V})[17,18]$.

\section{Reliability}

There was no significant difference in SLR range of motion and excellent reliability between trials for Phase 1 $\left(\mathrm{p}=0.332-0.899 ; \mathrm{ICC}_{2,1}: 0.96-0.99\right)$ and Phase $2(\mathrm{p}=0.356$ $\left.0.839 ; \mathrm{ICC}_{2,1}: 0.94-0.97\right)$ so both groups were combined for the remainder of the reliability analysis. For repeated testing, ICCs $\mathrm{IC}_{2,1}$ were 0.97 (95\% CI: 0.94, 0.98) for left PF/SLR with $95 \%$ limits of agreement between $-10.5^{\circ}$ and $9.9^{\circ}$. With right PF/SLR, the ICC was 0.96 (95\% CI: $0.93,0.98$ ) with $95 \%$ limits of agreement between $-12.0^{\circ}$ and $11.1^{\circ}$. For left DF/SLR the ICC was 0.98 (95\% CI: $0.96,0.99)$ with the $95 \%$ limits of agreement between $-8.4^{\circ}$ and $8.8^{\circ}$. For right DF/SLR the ICC was $0.96(95 \%$ CI: $0.93,0.98)$ with the $95 \%$ limits of agreement between $-9.8^{\circ}$ and $11.9^{\circ}$.

\section{Ankle positioning during SLR}

During Phase 2, there was no difference between the initial ankle position between trials (Table 2) with good reliability $\left(\mathrm{ICC}_{2,1}: 0.78-0.89\right)$, indicating that the ankle was positioned consistently between trials. The total ankle range of motion utilized in Phase 2 (30.0-32.3 $)$ with manual stabilization (Table 2) was similar to that utilized in Phase $1\left(30^{\circ}\right)$ with fixation using the brace.

\section{SLR range of motion}

The SLR range of motion ranged from approximately $15^{\circ}$ to over $90^{\circ}$ (Figure 1 ). When examining group means for SLR range of motion, there was no difference between the right and left limbs during either PF/SLR and DF/SLR (Table 2). In fact, the group average of both phases appears nearly identical between limbs $\left(<1^{\circ}\right.$ difference). However, there is a significant difference when looking at the average intra-individual, inter-limb differences (Figure 2). For both phases combined, the interlimb difference with PF/SLR averaged 5.0 $\left(3.5^{\circ} \mathrm{SD}\right.$; 95\% CI: $\left.3.8^{\circ}, 6.1^{\circ}\right)$ and $4.1^{\circ}\left(3.2^{\circ} \mathrm{SD}\right.$; 95\%CI: $\left.3.1^{\circ}, 5.1^{\circ}\right)$ with DF/SLR (Figure 2). Based upon the upper limit of

Table 1 Participant Demographics and correlations

\begin{tabular}{|c|c|c|c|c|c|c|c|c|c|}
\hline & \multicolumn{2}{|c|}{ Subgroups } & \multirow{3}{*}{$\begin{array}{c}\text { Combined } \\
\text { groups }\end{array}$} & \multicolumn{6}{|c|}{ Correlations (compared to combined groups) } \\
\hline & \multirow[b]{2}{*}{ Phase 1} & \multirow[b]{2}{*}{ Phase 2} & & \multicolumn{3}{|c|}{ PF/SLR range of motion } & \multicolumn{3}{|c|}{ DF/SLR range of motion } \\
\hline & & & & Left & Right & $\begin{array}{l}\text { Inter limb } \\
\text { difference }\end{array}$ & Left & Right & $\begin{array}{l}\text { Inter limb } \\
\text { difference }\end{array}$ \\
\hline Age (years) & $36.9 \pm 12.8 *$ & $29.4 \pm 7.3 *$ & $33.1 \pm 11.0$ & 0.18 & 0.23 & 0.02 & 0.21 & 0.28 & -0.14 \\
\hline Height (m) & $1.7 \pm 0.1$ & $1.7 \pm 0.1$ & $1.7 \pm 0.1$ & -0.18 & -0.15 & -0.25 & -0.14 & -0.09 & -0.04 \\
\hline Weight (kg) & $69.9 \pm 15.9$ & $68.3 \pm 15.1$ & $69.1 \pm 15.3$ & $-0.47+$ & $-0.52+$ & -0.18 & $-0.42+$ & $-0.40+$ & -0.04 \\
\hline $\mathrm{BMI}\left(\mathrm{kg} / \mathrm{m}^{2}\right)$ & $24.9 \pm 4.4$ & $25.1 \pm 5.5$ & $25.0 \pm 4.9$ & $-0.45+$ & $-0.52+$ & -0.09 & $-0.41+$ & $-0.42+$ & -0.05 \\
\hline Sex (\% female) & $85 \%$ & $70 \%$ & $77.5 \%$ & $0.40+$ & $0.42+$ & 0.06 & $0.42+$ & $0.40+$ & -0.09 \\
\hline Hand dominance (\% right) & $70 \%$ & $80 \%$ & $75 \%$ & -0.22 & -0.27 & -0.04 & -0.23 & -0.23 & -0.05 \\
\hline VPT (V) & $7.8 \pm 3.1$ & $6.4 \pm 1.9$ & $7.1 \pm 2.6$ & 0.06 & 0.11 & 0.02 & 0.13 & 0.18 & -0.06 \\
\hline MBQ (total score) & $9.5 \pm 1.2 *$ & $8.6 \pm 1.5 *$ & $9.0 \pm 1.4$ & $0.50+$ & $0.57+$ & 0.14 & $0.51+$ & $0.52+$ & 0.18 \\
\hline $\begin{array}{l}B M I=\text { body mass index. } \\
V P T=\text { vibration perception thr } \\
\text { sensation and normal being b } \\
M B Q=\text { Modified Baecke Quest } \\
\text { self-reported activity levels. } \\
P F=\text { plantar flexion. } \\
D F=\text { dorsiflexion. } \\
S L R=\text { straight leg raise. } \\
\text { * = statistically significant diffe } \\
\dagger=\text { statistically significant corr } \\
\text { Alpha set at } 0.05 \text {. }\end{array}$ & $\begin{array}{l}\text { hold at distal pa } \\
\text { ween } 0-15 \mathrm{~V} \text {. } \\
\text { nnaire; 3-15 poin } \\
\text { nce between grc } \\
\text { ation coefficient }\end{array}$ & $\begin{array}{l}\text { of halluces; 0-5 } \\
\text { scale with high }\end{array}$ & $\begin{array}{l}\text { scale with high } \\
\text { numbers indica }\end{array}$ & numbers & $\begin{array}{l}\text { dicating } p \\
\text { f-reported }\end{array}$ & rer sensation & d lower n & bers indic & ing better \\
\hline
\end{tabular}


Table 2 SLR range of motion

\begin{tabular}{|c|c|c|c|c|c|c|}
\hline & \multicolumn{3}{|c|}{$\mathrm{PF} / \mathrm{SLR}$} & \multicolumn{3}{|c|}{$\mathrm{DF} / \mathrm{SLR}$} \\
\hline & Left & Right & $p$ value & Left & Right & $p$ value \\
\hline \multicolumn{7}{|c|}{ Hip elevation angle } \\
\hline Phase 1 & $59.7^{\circ}\left(18.5^{\circ}\right)$ & $60.2^{\circ}\left(18.7^{\circ}\right)$ & 0.693 & $51.5^{\circ}\left(17.6^{\circ}\right)$ & $52.9^{\circ}\left(18.3^{\circ}\right)$ & 0.219 \\
\hline Phase 2 & $54.5^{\circ}\left(14.8^{\circ}\right)$ & $53.3^{\circ}\left(15.3^{\circ}\right)$ & 0.440 & $45.6^{\circ}\left(14.4^{\circ}\right)$ & $44.9^{\circ}\left(13.7^{\circ}\right)$ & 0.611 \\
\hline Both Phases & $57.1^{\circ}\left(16.8^{\circ}\right)$ & $56.7^{\circ}\left(17.2^{\circ}\right)$ & 0.752 & $48.5^{\circ}\left(16.1^{\circ}\right)$ & $48.9^{\circ}\left(16.4^{\circ}\right)$ & 0.692 \\
\hline \multicolumn{7}{|c|}{ Ankle position (Phase 2 only) } \\
\hline Initial position & $33.8^{\circ}\left(9.8^{\circ}\right) \mathrm{PF}$ & $32.0^{\circ}\left(12.8^{\circ}\right) \mathrm{PF}$ & 0.599 & $2.0^{\circ}\left(6.3^{\circ}\right) \mathrm{PF}$ & $1.1^{\circ}\left(6.3^{\circ}\right) \mathrm{PF}$ & 0.750 \\
\hline Ending position & $31.1^{\circ}\left(9.5^{\circ}\right) \mathrm{PF}$ & $30.5^{\circ}\left(12.4^{\circ}\right) \mathrm{PF}$ & 0.821 & $3.4^{\circ}\left(7.0^{\circ}\right) \mathrm{PF}$ & $0.8^{\circ}\left(6.9^{\circ}\right) \mathrm{DF}$ & 0.224 \\
\hline
\end{tabular}

$P F=$ plantar flexion

$D F=$ dorsiflexion.

$S L R=$ straight leg raise.

tolerance interval calculations, we can be $95 \%$ sure that $90 \%$ of the general population would have inter-limb differences of no greater than $10.9^{\circ}$ for PF/SLR and $9.4^{\circ}$ for DF/SLR.

One participant was an outlier as their inter-limb difference was greater than 3 SDs above the mean during PF/SLR $\left(17.0^{\circ}\right)$ and during DF/SLR $\left(16.5^{\circ}\right)$. Further evaluation of this participant's data revealed no evidence of confounding variables such as asymmetrical recreational activities, previous injuries or surgeries and confirmed that they were asymptomatic and had a normal neurological exam. For comparison purposes, when this individual was removed from the data analysis, the interlimb difference was not remarkably different with $4.7^{\circ}$ $\left(2.9^{\circ} \mathrm{SD} ; 95 \% \mathrm{CI}: 3.7^{\circ}, 5.6^{\circ}\right)$ during PF/SLR and $3.8^{\circ}\left(2.5^{\circ}\right.$ SD; $\left.95 \% C I: 3.0^{\circ}, 4.6^{\circ}\right)$ during DF/SLR. The upper limit of tolerance interval also was similar with this individual removed; we can be $95 \%$ sure that $90 \%$ of the general population would have inter-limb differences of no greater than $9.6^{\circ}$ for PF/SLR and $8.0^{\circ}$ for DF/SLR.

Overall SLR range of motion was moderately correlated with several demographic characteristics of the participants (Table 1). Specifically, bilateral SLR range of motion during both PF/SLR and DF/SLR had a

\section{A}

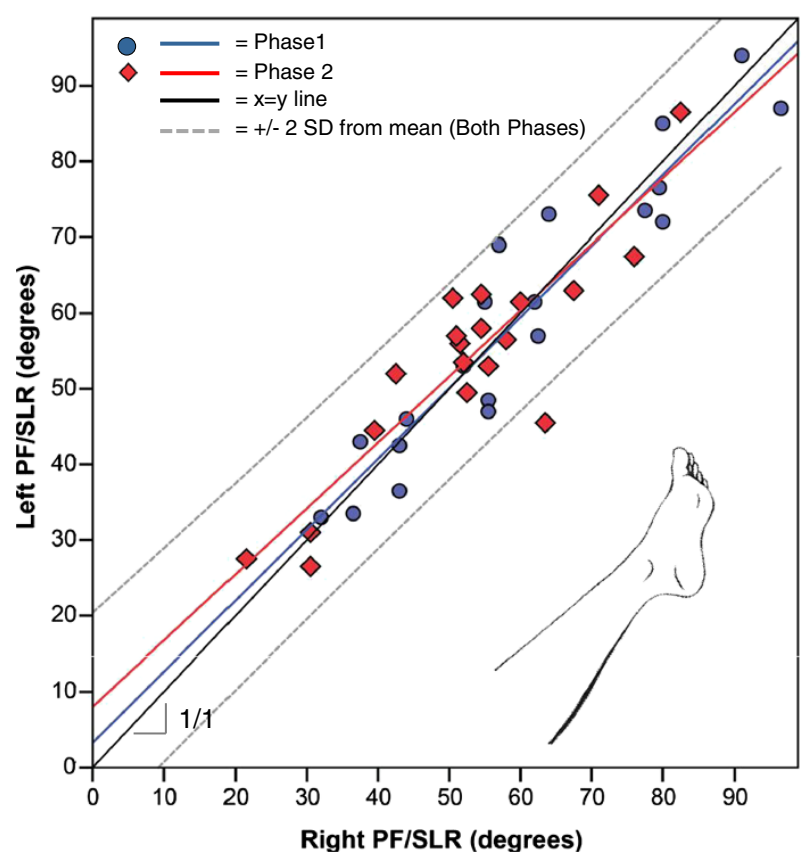

B

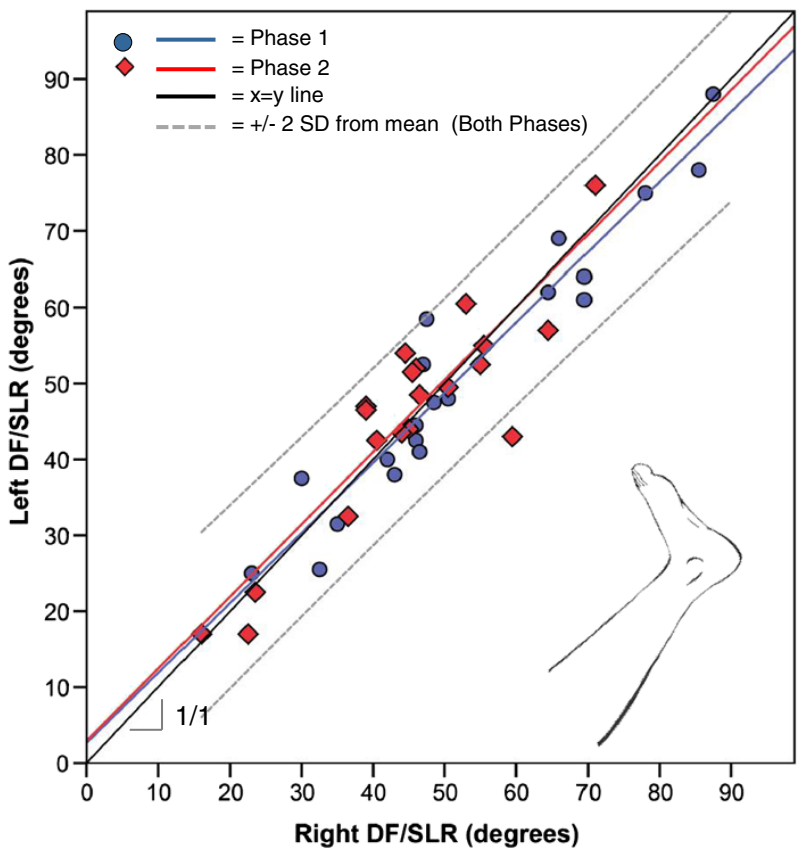

Figure 1 Overall SLR range of motion. SLR range of motion during PF/SLR (A) and DF/SLR (B) for the right ( $x$-axis) and left ( $y$-axis) are presented in degrees for Phase 1 (red) and Phase 2 (blue) including best fit lines for each phase. Black line represents the absolute $y=x$ condition as indicated by the $1 / 1$ slope. The grey dotted lines represent \pm 2 standard deviations from the mean for Both Phases. Data points above the $y=x$ line are indicative of more SLR range of motion on the left limb and those below this line indicate more on the right limb. 


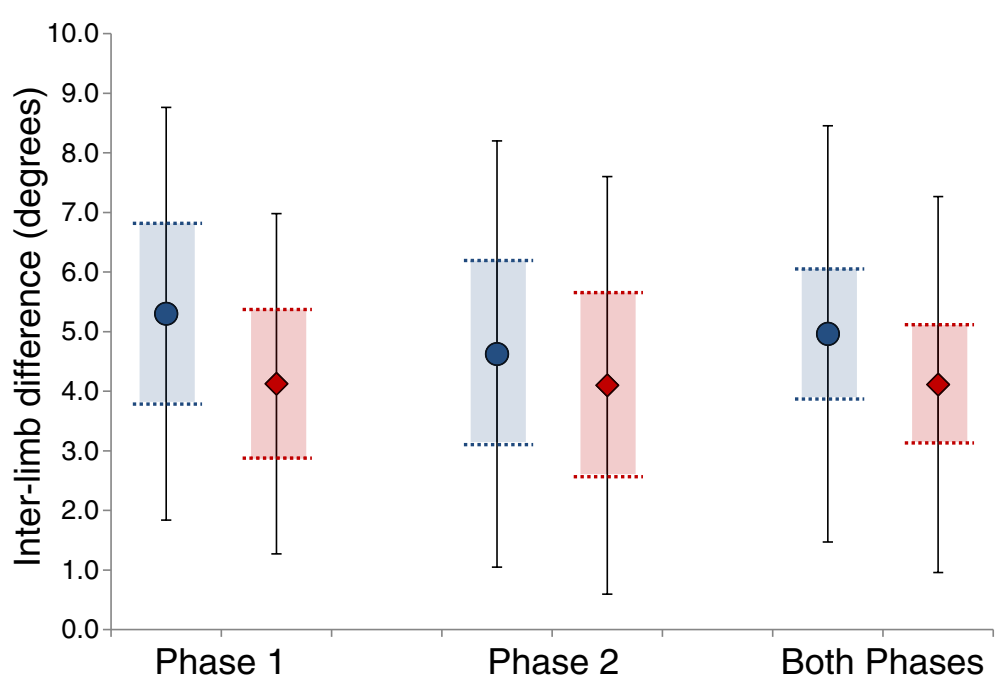

Figure 2 Intra-individual, inter-limb differences during SLR testing. Mean inter-limb differences are presented in degrees for Phase 1, Phase 2 and Both Phases for PF/SLR (blue) and DF/SLR (red). Solid black error bars represent standard deviations. Horizontal dotted lines and shaded area represent the $95 \%$ confidence interval for the mean inter-limb difference for PF/SLR (blue) and DF/SLR (red).

moderate negative correlation with weight and BMI such that higher weight or BMI was associated with less SLR range of motion. As expected, sex was moderately associated with SLR range of motion indicating that females had more SLR range of motion than men. Lastly, there was a moderate positive correlation with self-reported activity level on the MBQ. This indicates that individuals that reported a higher activity level had more SLR range of motion. There were no associations found between the SLR range of motion and age, height, hand dominance or VPT. In contrast, inter-limb difference was not significantly correlated with any demographic characteristic (Table 1).

\section{Discussion}

SLR neurodynamic testing range of motion is highly variable, ranging from approximately $15^{\circ}$ to over $90^{\circ}$ with a moderate association with multiple demographic characteristics, such as sex, weight, BMI and activity level. Specifically, heavier and less active individuals had lower SLR range of motion bilaterally compared to more active individuals who weighed less, just as women had more SLR range of motion bilaterally compared to men. The correlations between these demographic characteristics and overall SLR range of motion were similar bilaterally suggesting that the influence of these factors is equivalent in each limb. Previous studies have found similar variability in SLR range of motion $[1,3,6,9]$ and that females have more SLR range of motion compared to men [23]. Establishing a cutoff for normal SLR range for motion is problematic with such a high degree of variability and with so many demographic characteristics related to mobility.
In contrast, variability in inter-limb differences was much smaller and was independent of these demographic factors. For purposes of generalizability to the greater population, we can use the upper limit of a tolerance interval. Based upon this calculation, we can be $95 \%$ certain that "normal" inter-limb differences would be no greater than $10.9^{\circ}$ for PF/SLR and $9.4^{\circ}$ for DF/SLR in $90 \%$ of the general population of healthy individuals. Findings above these ranges could be considered non-normal and potentially important if found in a patient experiencing unilateral lower extremity pain. Further validation for this threshold comes from two previous studies that examined the inter-limb difference in symptomatic individuals. One study found an average of $12^{\circ}$ less mobility on the symptomatic side in people with low back pain with or without lower extremity pain with a positive SLR test [4]. The other study found an average of $30^{\circ}\left(\mathrm{SD} 10^{\circ}\right.$; range $10^{\circ}$ to $55^{\circ}$ ) less range of motion in people with unilateral lumbar radiculopathy [24].

Utilizing intra-individual, inter-limb differences as the normative standard provides added value because this measurement is independent of various demographic characteristics that commonly impact overall SLR range of motion. In contrast, comparing group means between limbs of healthy, asymptomatic individuals to establish the normative standard for asymmetry in SLR range of motion does not tell the whole story of normal responses to SLR testing. If equal percentages of individuals have greater SLR range of motion on the left (above the $y=x$ line in Figure 1) as do have on the right (below the $y=x$ line in Figure 1), the group averages will equal out and appear to be no different. In fact, we found that considerable intra-individual asymmetries can be present even in healthy, asymptomatic individuals 
(Figure 2) despite nearly identical group means (Table 2). This is consistent with a previous study where greater than $5^{\circ}$ inter-limb differences in ankle range of motion has been documented despite no difference in group mean comparisons [14]. Clinically, intra-individual, inter-limb comparisons are valuable to help determine if neurodynamic involvement is present, which reinforces the need for normative values for this inter-limb difference. Recently, mean inter-limb differences of $7^{\circ}\left(6.6^{\circ} \mathrm{SD}\right)$ between the dominant and non-dominant limb were documented during upper limb neurodynamic testing [15]. While a threshold level was not presented in this study, one can be calculated from their data using a similar tolerance level upper limit such that we could be $95 \%$ certain that $90 \%$ of healthy individuals would have no more than a $18.4^{\circ}$ interlimb difference during upper limb neurodynamic testing. This range of "normal" inter-limb differences is higher than in the SLR. We speculate that this difference reflects how asymmetrical use of the upper limbs is more common than for the lower limbs, but further research is necessary to substantiate this hypothesized rationale for the differences noted.

Phase 1 aimed to control the confounding variable of ankle positioning by strict fixation of the ankle position as has been done in previous studies $[1-3,6]$. It is equally important to test the reliability and validity of manual fixation of ankle positioning during SLR testing, as was the aim of Phase 2. Previous research has suggested that ankle dorsiflexion to $10^{\circ}$ with the knee in full extension and during SLR testing is difficult to achieve and dorsiflexion may be limited to only $4.3-4.8^{\circ}$ (SDs: $3.6-4.8^{\circ}$ ) in this position $[6,19]$. For this reason, a neutral ankle position was targeted with DF/SLR in the present study. Repeatability of ankle positioning had good reliability $\left(\mathrm{ICC}_{2,1}: 0.78-0.89\right)$, but tended to be in $1.1^{\circ}$ to $2.0^{\circ}$ degrees shy of neutral dorsiflexion at the beginning of testing. On average, the ankle position changed by between $1.4^{\circ}$ and $2.7^{\circ}$ from the beginning to the end of SLR testing. This suggests that there was a slight shift in ankle position during manual fixation of the ankle, but that the change averaged less than $3^{\circ}$ and represents a potential confounding variable that may have influenced the outcome measures. Since there were no significant differences in inter-limb measurements between test phases (Figure 2) and reliability of measuring SLR range of motion was equivalent between phases, the threat to the overall study conclusion is minimal.

The question remains as to why healthy, asymptomatic individuals are not perfectly symmetrical. It is unlikely that sub-clinical nerve injuries are responsible for the asymmetries documented, as all participants had normal lower extremity segmental neurological exams and quantitative sensory testing within normal ranges. Despite considerable efforts to exclude individuals with injuries to the musculoskeletal system, it is possible that some individuals had sub-clinical injuries that were not apparent at the time of enrollment. In the current study, variability in individual activity levels on the MBQ was considerable. According to these results, recreational activities ranged from no primary mode of exercise to running, biking, weight training and participating in group exercise classes. Habitual asymmetrical use of the limbs during daily function and recreation may create asymmetries in the tolerance of the neural tissues to movement. There is considerable evidence that habitual use of our limbs is not symmetrical during activities such as gait initiation [25], walking [26,27], turning [28], jumping [29-31], kicking [32], and crossing our legs [33]. While $85 \%$ of participants in the present study were right hand dominant (for writing) which is similar to proportions presented in previous literature, [34] a limitation to the present study is that lower limb dominance was not characterized in these individuals. Previous literature using various methods for determining limb dominance has shown a strong association between being right hand dominant and being right foot dominant (75.5\%-93.5\%), with a slightly lower association between left hand and foot dominance (56.9-79.4\%) [34-36]. Lower limb dominance may have influenced the magnitude and direction of inter-limb asymmetries found in this study and further research is necessary to characterize the specific effects of lower limb dominance and asymmetrical activities on SLR range of motion.

Additional limitations include the small number of male participants, as equal distribution of men and women were not sought in this sample of convenience. It should be noted that the impact of sex that has been demonstrated in previous studies [23] was still evident in the present study despite unequal numbers of males and females. We did not account for the menstrual cycle in women participants, nor did we have participants perform a warm up prior to testing which are additional limitations to the present study, although it is hypothesized that the effect on SLR range of motion would be equal bilaterally and thus not affect inter-limb differences. Additionally, the high reliability demonstrated in the present study is limited to intra-rater, intra-session and cannot be extrapolated to comparisons between raters or between sessions measurements. Lastly, it is possible that small but clinically relevant correlations exist between demographic characteristics and range of motion measures that we were unable to detect due to inadequate power of the present study to detect correlations of 0.35 or less.

\section{Conclusion}

Overall SLR neurodynamic testing range of motion is quite variable and tends to be greater in women, in 
those that are more active and in those that weigh less with a lower BMI. Inter-limb differences should be expected during SLR testing in healthy, asymptomatic individuals, but these asymmetries do not seem to be affected by the same demographic characteristics that influence overall SLR range of motion. Inter-limb differences of $11^{\circ}$ or greater are outside of the normal range and thus may be valuable for comparisons to patients experiencing unilateral pain.

\section{Abbreviations}

BMI: Body mass index $\left(\mathrm{kg} / \mathrm{m}^{2}\right)$; SLR: Straight leg raise; PF/SLR: Straight leg raise performed with the ankle in plantar flexion; DF/SLR: Straight leg raise performed with the ankle in dorsiflexion; MBQ: Modified Baecke Questionnaire; VPT: Vibration perception threshold; SD: Standard deviation; Cl: Confidence interval; ICC: Intraclass correlation coefficient.

\section{Competing interests}

The authors declare that they have no competing interests and verify that there is no financial affiliation with any product presented in this manuscript.

\section{Authors' contributions}

BSB conceived, designed and implemented the study and contributed to writing the manuscript. PSV implemented the study, assisted with analysis and contributed to writing the manuscript. Both authors read and approved the final manuscript.

\section{Acknowledgements}

The authors would like to thank Erin Carlson, Alissa Lamb, Allison Legakis, and Leanna Stracco for their assistance with data collection and analysis and Robert Nee for his assistance with manuscript editing and statistical guidance regarding generalization of the results to the larger population by use of tolerance intervals. This project was supported in part by a Faculty Research Incentives Award from Samuel Merritt University awarded to BSB. The funding body had no role in the study design, data collection, analysis, interpretation of data, manuscript writing or submission for publication.

\section{Author details}

${ }^{1}$ Department of Physical Therapy, Samuel Merritt University, 450 30th Street, Oakland, CA 94609, USA. ${ }^{2}$ Kaiser Permanente, 975 Sereno Drive, Vallejo, CA 94589, USA.

Received: 29 October 2012 Accepted: 5 December 2012 Published: 10 December 2012

\section{References}

1. Boyd BS, Wanek L, Gray AT, Topp KS: Mechanosensitivity of the lower extremity nervous system during straight leg raise neurodynamic testing in healthy individuals. J Ortho Sports Phys Ther 2009, 39:780-790.

2. Boyd BS, Wanek L, Gray AT, Topp KS: Mechanosensitivity during lower extremity neurodynamic testing is diminished in individuals with Type 2 Diabetes Mellitus and peripheral neuropathy: a cross sectional study. BMC Neurol 2010, 10:75.

3. Coppieters M, Kurz K, Mortensen T, Richards N, Skaret I, McLaughlin L, Hodges $P$ : The impact of neurodynamic testing on the perception of experimentally induced muscle pain. Man Ther 2005, 10:52-60.

4. Walsh J, Hall T: Agreement and correlation between the straight leg raise and slump tests in subjects with leg pain. J Manipulative Physiol Ther 2009, 32:184-192.

5. Nee R, Butler DS: Management of peripheral neuropathic pain: Integrating neurobiology, neurodynamics, and clinical evidence. Phys Ther Sport 2006, 7:36-49.

6. Gajdosik RL, LeVeau BF, Bohannon RW: Effects of ankle dorsiflexion on active and passive unilateral straight leg raising. Phys Ther 1985 65:1478-1482.

7. Boyd BS: Measurement properties of a hand-held inclinometer during straight leg raise neurodynamic testing. Physiotherapy 2012, 98:174-179.
8. Youdas JW, Krause DA, Hollman JH, Harmsen WS, Laskowski E: The influence of gender and age on hamstring muscle length in healthy adults. J Orthop Sports Phys Ther 2005, 35:246-252.

9. Hsieh CY, Walker JM, Gillis K: Straight-leg-raising test. Comparison of three instruments. Phys Ther 1983, 63:1429-1433.

10. James B, Parker AW: Active and passive mobility of lower limb joints in elderly men and women. Am J Phys Med Rehabil 1989, 68:162-167.

11. Soucie JM, Wang C, Forsyth A, Funk S, Denny M, Roach KE, Boone D: Range of motion measurements: reference values and a database for comparison studies. Haemophilia 2011, 17:500-507.

12. Nolan M, Nitz J, Choy NL, Illing S: Age-related changes in musculoskeletal function, balance and mobility measures in men aged 30-80 years. Aging Male 2010, 13:194-201

13. Macedo LG, Magee DJ: Differences in range of motion between dominant and nondominant sides of upper and lower extremities. J Manipulative Physiol Ther 2008, 31:577-582.

14. Ferrario VF, Turci M, Lovecchio N, Shirai YF, Sforza C: Asymmetry of the active nonweightbearing foot and ankle range of motion for dorsiflexion-plantar flexion and its coupled movements in adults. Clin Anat 2007, 20:834-842

15. Boyd BS: Common Interlimb Asymmetries and Neurogenic Responses during Upper Limb Neurodynamic Testing: Implications for Test Interpretation. J Hand Ther 2012, 25:56-64.

16. Baecke JA, Burema J, Frijters JE: A short questionnaire for the measurement of habitual physical activity in epidemiological studies. Am J Clin Nutr 1982, 36:936-942.

17. Garrow AP, Boulton AJ: Vibration perception threshold-a valuable assessment of neural dysfunction in people with diabetes. Diabetes Metab Res Rev 2006, 22:411-419.

18. Kastenbauer T, Sauseng S, Sokol G, Auinger M, Irsigler K: A prospective study of predictors for foot ulceration in type 2 diabetes. $J$ Am Podiatr Med Assoc 2001, 91:343-350.

19. Kwon OY, Minor SD, Maluf KS, Mueller MJ: Comparison of muscle activity during walking in subjects with and without diabetic neuropathy. Gait Posture 2003, 18:105-113.

20. Hopkins WG: Measures of reliability in sports medicine and science. Sports Med 2000, 30:1-15.

21. Portney LG, Watkins MP: Foundations of Clinical Research: Applications to Practice. 3rd edition. Upper Saddle River, New Jersey: Prentice-Hall International (UK) Limited, London; 2008.

22. NIST/SEMATECH: Tolerance intervals for a normal distribution. Engineering Statistics Handbook. 2012 [http://www.itl.nist.gov/div898/handbook/prc/ section2/prc263.htm].

23. Herrington L, Bendix K, Cornwell C, Fielden N, Hankey K: What is the normal response to structural differentiation within the slump and straight leg raise tests? Man Ther 2008, 13:289-294.

24. Stretanski MF: H-reflex latency and nerve root tension sign correlation in fluoroscopically guided, contrast-confirmed, translaminar lumbar epidural steroid-bupivacaine injections. Arch Phys Med Rehabil 2004, 85:1479-1482

25. Dessery Y, Barbier F, Gillet C, Corbeil P: Does lower limb preference influence gait initiation? Gait Posture 2011, 33:550-555.

26. Nagano H, Begg RK, Sparrow WA, Taylor S: Ageing and limb dominance effects on foot-ground clearance during treadmill and overground walking. Clin Biomech (Bristol, Avon) 2011, 26:962-968.

27. Senden R, Heyligers IC, Meijer K, Savelberg H, Grimm B: Acceleration-based motion analysis as a tool for rehabilitation: exploration in simulated functional knee limited walking conditions. Am J Phys Med Rehabil 2011, 90:226-232

28. Strike SC, Taylor MJ: The temporal-spatial and ground reaction impulses of turning gait: is turning symmetrical? Gait Posture 2009, 29:597-602.

29. Stephens TM 2nd, Lawson BR, Reiser RF 2nd: Bilateral asymmetries in max effort single-leg vertical jumps. Biomed Sci Instrum 2005, 41:317-322.

30. Stephens TM 2nd, Lawson BR, De Voe DE, Reiser RF 2nd: Gender and bilateral differences in single-leg countermovement jump performance with comparison to a double-leg jump. J Appl Biomech 2007, 23:190-202.

31. Pappas E, Carpes FP: Lower extremity kinematic asymmetry in male and female athletes performing jump-landing tasks. J Sci Med Sport 2012, 15:87-92.

32. McLean BD, Tumilty DM: Left-right asymmetry in two types of soccer kick. Br J Sports Med 1993, 27:260-262. 
33. Reiss M: Leg-crossing: incidence and inheritance. Neuropsychologia 1994, 32:747-750.

34. Dittmar M: Functional and postural lateral preferences in humans: interrelations and life-span age differences. Hum Biol 2002, 74:569-585.

35. Barut C, Ozer CM, Sevinc O, Gumus M, Yunten Z: Relationships between hand and foot preferences. Int J Neurosci 2007, 117:177-185.

36. Kumar S, Misra I, Suman S, Suar D, Mandal MK: Interrelationship of limb dominance and sensory function across age. Int I Neurosci 2010, 120:110-114.

doi:10.1186/1471-2474-13-245

Cite this article as: Boyd and Villa: Normal inter-limb differences during the straight leg raise neurodynamic test: a cross sectional study. BMC Musculoskeletal Disorders 2012 13:245.

\section{Submit your next manuscript to BioMed Central and take full advantage of:}

- Convenient online submission

- Thorough peer review

- No space constraints or color figure charges

- Immediate publication on acceptance

- Inclusion in PubMed, CAS, Scopus and Google Scholar

- Research which is freely available for redistribution 